\title{
A Phase Cell Approach to Yang-Mills Theory V. Analysis of a Chunk ${ }^{\star}$
}

\author{
P. Federbush \\ Department of Mathematics, University of Michigan, Ann Arbor, MI 48109, USA
}

\begin{abstract}
In the present formalism the Yang-Mills field is constructed as a "non-linear sum" of excitations, small field excitations, the modes, and large field excitations, the chunks. The chunk excitations, herein studied, are each described by a finite number of group element variables. The continuum field associated to the excitation in general has point gauge singularities (arising from the non-trivial $\pi_{3}(G)$ ). We find estimates for plaquette assignments, edge assignments, and the smoothness of edge assignments, at all scales. The central conceptual motor in our constructions and estimates is a split up of the field at each length scale, locally, into a pure gauge field, and a deviation field. An example is presented establishing the general inevitability of gauge singularities, as a consequence of fall off requirements on the continuum field of an excitation.
\end{abstract}

\section{Introduction}

This is the fifth in a series of papers developing a treatment of the finite volume Euclidean Yang-Mills quantum field theory in four dimensions. To read this paper familiarity with the Papers I and IV in this series is assumed. (We refer to papers in this series with Roman numerals.) Not all of the material in these two papers will be of present interest to us, in particular the interesting mechanism of "gauge invariant coupling" introduced in IV will not be herein used. Of most interest will be Sects. 8-11 and the appendices of IV; we will detail references in the course of the paper. The abstract and introduction of IV serve also as a partial introduction to this paper (and the overall program) and should now be read; we in this introduction complement the introduction of IV. We begin by addressing two questions we have been often asked.

Does the procedure use a lattice formalism, or work with continuum fields? In the old days one asked, "is the electron a particle or a wave?" One there dealt with a "particle-wave duality." We deal with a "lattice-continuum duality," as

* This work was supported in part by the National Science Foundation under Grant No. PHY-87-01329 
introduced in $I$. We work with continuum fields, but describe the continuum fields by group element variables, conveniently related to group elements living on bonds of lattices. Our lattices are a mathematical construction to associate variables to the continuum field - a nonlinear association which we regard as realization of the epigram "The Yang-Mills theory is an essentially non-linear field theory." Loop variables constructed from the group elements associated to lattice loops are gauge invariant variables associated to the field (this is not true in Balaban's work), and the existence of their expectation values will have been directly constructed once the phase cell expansion is established.

The basic problem studied in this paper involves the problem of determining a continuum field that approximately minimizes the continuum action subject to the constraint of some of its variables being fixed-those living on some of the lattices. This has lead to a second question we have been frequently asked. Why is it sufficient to only approximately minimize the action? One may also ask why the Chinese no longer bind their daughters' feet. One does not define variables for the $\phi_{3}^{4}$ field theory by exactly minimizing the full action-- though presumably one could. It is true that Balaban works with configurations that exactly minimize the action (on a fine lattice) subject to variables being fixed on a coarse lattice-we address the same problem by using "gauge invariant coupling" (the problem of avoiding coupling to large scale variables through the $A$ field instead of the $F$ field).

In this paper we study the continuum field associated to a chunk. A chunk is precisely defined in Sect. 8 of IV. For a given $S-L$ configuration one has specified at all levels which plaquettes, in $\mathscr{P}=\bigcup_{r} \mathscr{P P}$, are $S$ and which are $L$. Loosely speaking, a chunk $E=\left\{e_{i}\right\}$ consists of a finite collection of edges $e_{i} \in \mathscr{E}=\bigcup_{r} \mathscr{E}^{r}$ such that each $e_{i}$ is "close" to an $L$ plaquette, and near it in level. The chunk "surrounds" each $L$-plaquette in it by $S$-plaquettes. Variables are specified on the edges of the chunk and one must find a continuum field sufficiently close to minimizing the action subject to being specified on these edges. One has much gauge freedom, in particular one may arbitrarily specify a gauge field, $h_{i}$, at all vertices not in the chunk. One must in addition choose the gauge to ensure sufficient smoothness in the resultant continuum field.

The continuum field is constructed by inductively giving its assignments to each lattice going down the scale (given the assignments to $\mathscr{L}^{r}$, the assignments to $\mathscr{L}^{r+1}$ are constructed). The gauge choice, on $\mathscr{L}^{r+1}$ is specified uniquely by specifying assignments to a maximal tree in each block in $\mathscr{L}^{r+1}$. This is "gauge interpolation," whose study is initiated in Sect. 11 of IV. (The gauge choice on $\mathscr{L}^{r+1}$ "sort of" interpolates the gauge choice on $\mathscr{L}^{r}$.) Once the gauge is determined on $\mathscr{L}^{r+1}$, the assignments to the remaining edges of $\mathscr{L}^{r+1}$ are made, "field interpolation" as specified in Sect. 10 of IV. The deviations from a pure gauge field are very small, and field interpolation uses local approximation by abelian fields and local minimization of the abelian action.

As the prototype problem studied in this paper we consider the following situation. We are given a configuration on the lattice $\mathscr{L}^{r}$, an assignment of group elements to oriented bonds of the lattice. We seek a continuum field, $A_{\mu}(x)$, associated to this configuration on $\mathscr{L}^{r}$, and minimizing the continuum action, 
$\int|F|^{2}$, subject to this constraint. The association of continuum fields to lattice configurations has been analyzed in I and VI. Because of gauge invariance such $A_{\mu}(x)$ are not unique; among the minimizing solutions we seek one with smoothness properties and fall off at infinity (if the configuration on $\mathscr{L}^{r}$ so does). In I and II this problem was solved precisely, in the case all assigned group elements on $\mathscr{L}^{\text {r }}$ lie in an abelian subgroup of the gauge group $G$, and are sufficiently close to the identity. Under these conditions one may work with $A_{\mu}(x)$ minimizing the abelian continuum action.

For chunks, large field excitations, the situation is much more complicated. The isolated chunk field, as made explicit in Sects. 8-11 of IV, is specified by a finite number of group element variables, living on a number of levels. In this introduction we restrict our discussion to the prototype problem, the heart of our analysis. As we will explain, we compromise the original problem, we only approximately minimize the continuum action. More important, we do not achieve full smoothness; point singularities will arise in the gauge field. We will prove that such singularities are necessary if fall off at infinity is demanded.

We first consider the special case when the assigned configuration on $\mathscr{L}^{\text {r }}$ is pure gauge. That is, to each edge $e=\overrightarrow{a b}$ in $\mathscr{L}^{r}$, we have the assignment $\Lambda^{-1}(a) \Lambda(b)$, where

$$
\Lambda: \mathscr{V}^{r} \rightarrow G,
$$

with $\mathscr{N}^{r}$ the vertices of $\mathscr{L}^{r}$. A pure gauge $A_{\mu}(x)$, i.e. one with $F_{\mu \nu}(x)=0$, associated to the assignment on $\mathscr{L}^{r}$ would clearly minimize the action-setting it to zero. If the mapping in (1.1) is extended from $\mathscr{V}^{r}$ to $R^{4}$, then such an $A_{\mu}(x)$ can be found by setting

$$
A_{\mu}(x)=\Lambda^{-1}(x) \partial_{\mu} \Lambda(x) .
$$

Such extensions are always possible.

In Appendix B we exhibit a pure gauge configuration on $\mathscr{L}^{r}$, which falls off at infinity, for which we can find an associated pure gauge $A_{\mu}(x)$ falling off at infinity, with a point singularity; but to which no pure gauge $A_{\mu}(x)$ is associated that is both everywhere continuous and has such fall off. This paradigm establishes the generic inexorability of gauge singularities as a concomitant of fall off requirements. In this example we deal with power law fall off, but we expect that examples can be constructed with exponential fall off (of necessity, not pure gauge).

We continue to discuss the case of a pure gauge configuration on $\mathscr{L}^{r}$. As alluded above the problem of finding action minimizing $A_{\mu}(x)$ is equivalent to the problem of extending $\Lambda$ from $\mathscr{V}^{r}$ to $R^{4}$. The procedure we follow to accomplish this is presented in Sect. 11 of IV. Two nearest neighbor vertices in $\mathscr{V}^{r}$ are viewed as the boundary $S^{0}$ of the straight line segment joining them. The mapping $\Lambda$ is extended from $S^{0}$ to this line segment. When $\Lambda$ has been extended over all such line segments similarly, one next considers a face, whose boundary, viewed as $S^{1}$, is made up of four such line segments. $A$ has been defined already on $S^{1}$, it is extended to the face. We do assume that $\pi_{1}(G)$ is trivial, so this extension may be continuous. We likewise extend from the boundary of volumes (=hyperfaces), viewed as $S^{2}$ 's, to hyperfaces. $\left(\pi_{2}(G)\right.$ is trivial.) Finally we extend from the boundary of hypercubes, 
$\sim S^{3}$, to the interior of the hypercubes. Here we may encounter maps from $S^{3}$ to $G$ that are homotopically non-trivial, and thus encounter the necessity of dealing with point gauge singularities. (There is no topological obstruction to the extension of maps into $G$ from $\mathscr{V}^{r}$ to $R^{4}$ which forces singularities. Our construction leads to singularities. However as the paradigm above illustrates, avoiding the singularities means one must sacrifice fall off at infinity. "One constructs a tube, on which $A_{\mu}(x)$ does not go to zero, pushing the singularity out to infinity.") The specific restrictions on extensions to yield smoothness are given in the Geometric Constructions of Sect. 11 of IV, analyzed in Theorems A.1, A.3, and A.4 of IV.

We now proceed to the general situation when the configuration on $\mathscr{L}^{r}$ need not be a pure gauge. $\mathscr{L}^{r}$ divides space into (hyper) cubes. We define a pure gauge field on each of these cubes separately. A maximal tree is selected of the edges in the boundary of a given cube. The group elements of the configuration on $\mathscr{L}^{r}$ restricted to the maximal tree may be viewed as pure gauge on the maximal tree, arising from a $\Lambda(x)$ restricted to the vertices of the cube. This $\Lambda$ may be extended from the vertices of the cube, throughout the whole cube, giving the local pure gauge field in the given cube. Such local pure gauge fields in each given cube, are chosen to match well with the pure gauge fields of the neighboring cubes - for pure gauge fields in $\mathscr{L}^{r}$ the matching may be chosen perfect as above. The matching is discussed in Sect. 11 of IV, and Theorem A.2 of IV. The discussion is further amplified in Appendix D herein. (We recommend reading Sects. 8-11 and Appendix A of IV, followed by Appendix D, before reading the body of this paper.)

We now discuss the configuration on $\mathscr{L}^{r+1}$ associated to the configuration on $\mathscr{L}^{r}$ by the developments in Sects. 10 and 11 of IV. Section 10 of IV specifies the configuration on $\mathscr{L}^{r+1}$ up to a gauge transformation - the configuration chosen arises from continuum fields that (locally) approximately minimize the continuum action. In Sect. 2 we derive properties of this field independent of the gauge choice, and likewise properties of the configuration on $\mathscr{L}^{s}, s>r$, derived by our inductive construction. In particular Theorem 2.2 yields estimates of the loose form

$$
\left|g_{\partial p}\right| \leqq c\left(\frac{l_{s}}{l_{r}}\right)^{2} \sup _{P \in \mathscr{P}^{r}}\left|g_{\partial p}\right|
$$

where $P$ is the plaquette in $\mathscr{L}^{s}$, and $\mathscr{P}^{r}$ is the set of plaquettes in $\mathscr{L}^{r}$.

Each edge $e$, in $\mathscr{L}^{r+1}$ may be viewed as living in one of the cubes we have discussed above, and so the edge may be given an assignment by the pure gauge field associated to the cube; we call this assignment $g^{G}(e)$. Section 3 studies the difference between the actual assignment to $e, g(e)$, and $g^{G}(e)$. Bounds are obtained on $\left\{g(e)-g^{G}(e) \mid\right.$. (Here we view the $g$ 's as unitary operators letting the bars represent operator norms. We also use bars for another purpose, by $|g|$ we mean $d(g, I d), d$ the invariant distance on $G$ arising from the invariant metric.) We have split the field locally into a pure gauge part, and a deviation. This type of analysis seems rather basic, and we expect it will have other applications-perhaps it already has been used by others which we do not know about.

Section 4 studies the $g^{G}$ themselves. This is the hardest section of the paper, and the prettiest. We feel the reader who fights through Appendix D and Sect. 4--these go together-will find the effort rewarding. Basically one is involved 
with how well or how badly the local pure gauge fields of neighboring cubes match. The first part of Appendix D deals with the abstract setup for measuring how well two sets of gauge assignments agree - such as the assignments by the $\Lambda(x)$ of two neighboring cubes to their region of intersection. Here we must remember $\Lambda(x)$ and $u \Lambda(x)$ yield the same field $A_{\mu}(x)$ (via (1.2)) if $u \in G$ is constant.

Section 5 and Appendix E study the problem of edge smoothing, as introduced at the end of Sect. 10 of IV. This is perhaps a technical matter, of less interest than the rest of the paper.

Finally we note Appendix $\mathrm{C}$ makes a few changes and additions to structures defined in previous papers.

\section{Plaquette Estimates}

Definition 2.1. Let $E$ be a chunk, and $\mathscr{E}(E)$ be the level $r$ edges of $E$, as defined in Sect. 8 of IV. (We may write $\mathscr{E}^{r}(E)=E \cap \mathscr{E}^{r}$.) For any point $x$ in $R^{4}$, and level $r^{\prime}$, we now define $r_{E}\left(x, r^{\prime}\right)$ by

$$
r_{E}\left(x, r^{\prime}\right)=\operatorname{Max}\left\{S_{E}(x) \cap\left[0, r^{\prime}\right]\right\}
$$

with

$$
S_{E}(x)=\left\{r, d\left(x, \mathscr{E}^{\mathscr{C}}(E)\right) \leqq C_{1 c} N l_{r}\right\} .
$$

If $r_{E}\left(x, r^{\prime}\right)$ is not defined, we say it is "empty." $r_{E}\left(x, r^{\prime}\right)$ is a sort of the level of chunk edges lying above the point $x$ viewed as living at level $r^{\prime}$.

Theorem 2.2. Let $p$ be a plaquette of level $r^{\prime}$ outside the chunk E. Let $x_{p}$ be any vertex of $p$, and abbreviate $r_{E}\left(x_{p}, r^{\prime}\right)$ as $\tilde{r}_{p}$. Then

$$
\left|g_{\partial p}\right| \leqq c a\left(\frac{l_{r^{\prime}}}{l_{r_{p}}}\right)^{2}
$$

or if $\tilde{r}_{p}$ is empty

$$
\left|g_{\partial p}\right|=0 .
$$

Theorem 2.3. Let $p$ and $p^{\prime}$ be parallel plaquettes of level $r^{\prime}$, whose distance, measured between corresponding vertices, satisfies

$$
d\left(p, p^{\prime}\right)<c l_{r^{\prime}-1} .
$$

We develop plaquette values as given in a radial gauge on the $\mathscr{L}^{\prime}$ lattice with center at distance $<c l_{r^{\prime}-1}$ from $p$ and $p^{\prime}$. We let $x_{p}$ be a vertex of $p$ and abbreviate $r_{E}\left(x_{p}, r^{\prime}\right)=\tilde{r}_{p}$. We then have

$$
\left|g_{\hat{o} p}-g_{\partial p^{\prime}}\right| \leqq c a\left(\frac{d\left(p, p^{\prime}\right)}{l_{\tilde{r}_{p}}}\right)^{1-\varepsilon}\left(\frac{l_{r^{\prime}}}{l_{\tilde{r}_{p}}}\right)^{2-\varepsilon} \text {. }
$$

Again if $\tilde{r}_{p}$ is empty all plaquette values are trivial.

The following subsection contains estimates that lead to a proof of these theorems, and are interesting in their own right.

Field Interpolation Estimates. We consider a given configuration at level $r$, and 
study the configuration at level $r+1$ determined by field interpolation via Sect. 10 of IV. We consider a point $z_{0}$ in $\mathscr{V}^{r+1}$ and points $z_{1}$ in $\mathscr{V}^{r}$ satisfying

$$
d\left(z_{i}, z_{0}\right)<c l_{r} .
$$

We assume for $p \in \mathscr{P}^{r}$

$$
\left|g_{\partial p}\right| \leqq M \leqq \bar{\varepsilon}
$$

for $p$ within distance $C_{1 c} l_{r}$ of $z_{0}$ (where all the action takes place). $C_{1 c}$ will eventually be picked proportional to $N$. We also assume if plaquette values are calculated in the radial axial gauge about any of the $z_{i}$ one has, for $p, p^{\prime}$ in $\mathscr{P P}$ (and within distance $C_{1 c} l_{\text {r }}$ of $z_{0}$ )

$$
\left|g_{\partial p}-g_{\partial p^{\prime}}\right| \leqq f M \text {. }
$$

Estimate 2.4. If $e$ is an edge in $\mathscr{E}$ (at distance less than $C_{1 c} l_{\mathrm{r}}$ from $z_{0}$ ) and the field is calculated in a radial gauge about one of the $z_{i}$ then

$$
|g(e)| \leqq c C_{1 c}^{2} M \text {. }
$$

(The $C_{1 c}^{2}$ could be improved to $C_{1 c}$. There are a number of such possible improvements we do not comment on in this section.)

We have used Lemma 5.5 of III and properties of a radial tree. From now on we no longer repeat the injunction of being at distance less than $C_{1 c} l_{r}$ from $z_{0}$, always assumed.

Estimate 2.5. For a $p$ in $\mathscr{P}$, we calculate $g_{\partial p}$ in radial gauges centered at $z_{i}$ and $z_{j}\left(g_{\partial p}^{(i)}\right.$ and $\left.g_{\partial p}^{(j)}\right)$, gauge transformations of each other, and find

$$
\left|g_{\partial p}^{(i)}-g_{\partial p}^{(j)}\right| \leqq c M C_{1 c}\left(C_{1 c}^{2} M\right) .
$$

We have noted that $c C_{1 c}\left(C_{1 c}^{2} M\right)$ is a bound on the size of $h$ 's needed to implement the gauge transformation. The following follows from Lemma A.1 (see (A.1)).

Estimate 2.6. For a given Lie Algebra direction, we calculate plaquette values in $\mathscr{P}^{r+1}$ via the construction in the paragraph containing (10.7) in IV, for any of the $z_{i}$ (playing the role of $v$ in IV). Call these $A_{z_{i}}(\partial p)$. One has

$$
\begin{aligned}
\left|A_{z_{i}}(\partial p)\right| & \leqq c\left(\frac{l_{r+1}}{l_{r}}\right)^{2} M+c\left(\frac{l_{r+1}}{l_{r}}\right)^{2} f\left(C_{1 c}^{2} M\right) C_{1 c}^{3} e^{-\gamma^{\prime} c_{1 c / 2}}, \\
& \leqq c\left(\frac{l_{r+1}}{l_{r}}\right)^{2} M .
\end{aligned}
$$

In the last term in (2.9) we note $f\left(c C_{1 c}^{2} M\right)$ as a bound on modification of plaquette values at sphere boundary caused by truncation of (10.7) of IV, and $c C_{1 c}^{3}$ as the sphere volume.

Estimate 2.7. We view $\dot{z}_{i}$ as a vertex in $\mathscr{V}^{r+1}$ (equaling $z_{i}$ in $\mathscr{V}^{r}$ ) and work in the radial gauge about $\dot{z}_{i}$ in $\mathscr{L}^{r+1}$. Then as in the paragraph containing (10.8) of IV (with $z_{i}, \dot{z}_{i}$ as $v, \dot{v}$ therein) we construct $A$ fields for edges $e \in \mathscr{E}^{r+1}$, denoting them 
as $A_{z_{\mathbf{i}}}(e)$. For $e$ within distance $c l_{r}$ of $z_{0}$ one has

$$
\left|A_{z_{i}}(e)\right| \leqq c\left(\frac{l_{r+1}}{l_{r}}\right)^{2} M N^{2}
$$

Estimate 2.8.

$$
\begin{aligned}
\left|A_{z_{i}}(\partial p)-A_{z_{j}}(\partial p)\right| & \leqq c\left(\frac{l_{r+1}}{l_{r}}\right)^{2} M\left[C_{1 c}\left(C_{1 c}^{2} M\right)+\left(C_{1 c}^{2} \cdot C_{1 c}^{3} \cdot f\right) e^{-\gamma^{\prime} C_{1 c} / 2}\right] \\
& \leqq a_{1}(N) M^{2}+(\mathrm{es})_{1} f M
\end{aligned}
$$

We have used Estimate 2.5 and Lemma A.1. For a number of estimates now we will be less specific about constants (as in going from (2.12) to (2.13)). (es) stands for a factor "exponentially small" in some variable $\left(C_{1 c}\right), M$ will also be very small, each dominating any power of $N$ that occurs.

Estimate 2.9. Let $\hat{A}_{z_{i}}(\partial p)$ be the plaquette variables constructed from the edge variables $A_{z_{i}}(e)$. And let $A_{z_{i}}^{T}(\partial p)$ be the plaquette variables whose Lie Algebra components are the $A_{z_{i}}(\partial p)$. (This may be confusing. The $A_{z_{i}}(\partial p)$ are components in the different Lie Algebra directions of a Lie Algebra element $A_{z_{i}}^{T}(\partial p)$. But these $A_{z_{i}}^{T}(\partial p)$ may not be the plaquette variables associated to the $A_{z_{i}}(e)$-they would be if all elements commuted.) One has

$$
\left|A_{z_{i}}^{T}(\partial p)-\hat{A}_{z_{i}}(\partial p)\right| \leqq K_{1}(N) M^{2} .
$$

In fact we are bounding this difference by $c\left|\operatorname{Sup}_{e}\right| A_{z_{i}}(e)||^{2}$ and using Estimate 2.7 . There now follows from this last estimate, and the size of $h$ 's needed to perform a gauge transformation, the next estimate.

Eestimation 2.10. We let $\hat{A}_{z_{j}}^{G_{i}}(\partial p)$ be the gauge transformation of $\hat{A}_{z_{j}}(\partial p)$ to the radial axial gauge about $\dot{z}_{i}$

$$
\left|\hat{A}_{z_{i}}(\partial p)-\hat{A}_{z_{j}}^{G_{i}}(\partial p)\right| \leqq a_{2}(N) M^{2}+(\mathrm{es})_{2} f M .
$$

We have compared plaquette values as computed by the two different constructions, one about $\dot{z}_{i}$, one about $\dot{z}_{j}$, as measured in the same gauge. We now compare edge assignments in the two different constructions, as compared in same gauge.

Estimate 2.11 .

$$
\left|A_{z_{i}}(e)-A_{z_{j}}^{G_{i}}(e)\right| \leqq a_{3}(N) M^{2}+(\mathrm{es})_{3} f M .
$$

This follows from the previous estimate (easily if we note that $M$ will be very small). This estimate, and the next, show we will be able to approximate the interpolated field plaquette values, obtained by averaging edge values arising from different $z_{i}$ constructions, by any one of these going into the average-for small $M$.

Estimate 2.12. Let $\delta A(e)$ be the change of the assignment to an edge $e \in \mathscr{E}_{A}^{r+1}$ required to satisfy averaging, then

$$
|\delta A(e)| \leqq a_{4}(N) M^{2}+(\mathrm{es})_{4} f M .
$$


There follows from Estimates 2.6, 2.9-2.12.

Estimate 2.13. For $p \in \mathscr{P}^{r+1}$,

$$
\left|g_{\hat{\partial p}}\right| \leqq c\left(\frac{l_{r+1}}{l_{r}}\right)^{2} M+a_{5}(N) M^{2}+(\mathrm{es})_{5} f M .
$$

There easily follows

Estimate 2.14. There are functions $N_{0}(\varepsilon)$ and $\bar{\varepsilon}_{0}(N)$ such that for any $\varepsilon>0$ one has for $p \in \mathscr{P}^{r+1}$,

$$
\left|g_{\partial p}\right| \leqq\left(\frac{l_{r+1}}{l_{r}}\right)^{2-\varepsilon} M
$$

if

$$
N \geqq N_{0}(\varepsilon) \text { and } \bar{\varepsilon} \leqq \bar{\varepsilon}_{0}(N) .
$$

(See (2.5) for definition of $\bar{\varepsilon}$.)

Similarly we can prove

Estimate 2.15. There are functions $N_{1}(\varepsilon)$ and $\bar{\varepsilon}_{1}(N)$ such that for any $\varepsilon>0$, and for $p, p^{\prime}$ in $\mathscr{P}^{r+1}$ satisfying

$$
d\left(p, p^{\prime}\right)<c l_{r},
$$

one has (working in a radial gauge in the $\mathscr{L}^{r+1}$ lattice with center $<c l_{r}$ from $p$ and $p^{\prime}$ )

$$
\left|g_{\partial p}-g_{\partial p^{\prime}}\right| \leqq \frac{d\left(p, p^{\prime}\right)}{l_{r}}\left(\frac{l_{r+1}}{l_{r}}\right)^{2-\varepsilon} M\left(f+M^{2 / 3}\right),
$$

if

$$
N \geqq N_{1}(\varepsilon) \text { and } \bar{\varepsilon} \leqq \bar{\varepsilon}_{1}(N) .
$$

There is nothing very special about the $2 / 3$ in (2.21), this may be any number satisfying $1 / 2<x<1$. The introduction of $A_{0}^{\alpha}$ and $A_{\delta}^{\alpha}$ and the corresponding construction involving (10.7) of IV were devised to yield our last estimate. (One uses in deriving (2.21) the fact that, working to linear order in the fields, if the plaquette assignments at level $r$ are all the same, this is also true at level $r+1$ for the construction of Sect. 10 of IV.).

Theorem 2.3 follows from Estimate 2.15 by induction down the levels. From Estimate 2.14 there similarly follows immediately our next result.

Estimate 2.16. Under the same conditions as in Theorem 2.2,

$$
\left|g_{\partial p}\right| \leqq c a\left(\frac{l_{r^{\prime}}}{l_{\hat{r}_{p}}}\right)^{2-\varepsilon},
$$

and likewise trivial if $\tilde{r}_{p}$ is empty.

We again consider two neighboring levels $r$ and $r+1$. We consider a level $r$ plaquette $P$, and set $M_{r}=\left|g_{\partial P}\right|$. We consider plaquettes at level $r+1$ within distance $c l_{r}$ of $P$ and for such we set

$$
\underset{p}{\operatorname{Max}}\left|g_{\partial_{p}}\right|=M_{r+1}, \quad\left|g_{\hat{\partial p_{i}}}-g_{\partial p_{j}}\right| \leqq \Delta M_{r+1},
$$


where in the last relation $\Delta M_{r+1}$ is a bound on differences of plaquette values measured in a local radial gauge. From (2.1) of III, Infinitesimal Field Averaging, we get the inequality (recalling $l_{r}=1 / N^{r}$ ),

$$
M_{r+1} \leqq \frac{M_{r}}{N^{2}}+c \Delta M_{r+1}+c(N) M_{r+1}^{2} .
$$

We use Theorem 2.3 and Estimate 2.16 to bound the second and third terms on the right side of (2.25), respectively. Inequality (2.25) may then be used inductively to establish Theorem 2.2 .

\section{Edge Assignment of Estimates}

We first place a very mild requirement on our maximal trees, and the arbitrary choices that enter Eq. (11.14) of IV.

Quadrant Localization of Maximal Trees, and Gauge Choices. There is a constant $c$ (independent of $N$ ), such that the following holds. We consider a block in $\mathscr{B}^{r}$ with base point $v$, and let $w$ be another point in the block. Then $t_{v w}$ is the path in the maximal tree connecting $v$ and $w$, made up of edges in $\mathscr{E}_{I}^{r}$. Let this sequence of edges be $e_{1}, e_{2}, \ldots, e_{s}$. Then we may partition this sequence into $\leqq c$ segments (cutting the path $t_{v w}$ into $\leqq c$ subpaths) such that in each of these segments assignments to the edges via (11.14) of IV come from a single $H_{i}$ (more properly $\phi_{i}$ ).

We note that this is a requirement on trees; they may not wander back and forth endlessly from one quadrant to the other. It is also a requirement on the selections going into (11.14) of IV; for a path living on the boundary of two hypercubes, $H_{1}$ and $H_{2}$, the selections may not for example be alternately taken from $H_{1}$ and $H_{2}$.

We now define an edge from a small field neighborhood, or an SFN-edge in $\mathscr{E}^{r+1}$ as an edge $e$ in $\mathscr{E}^{r+1}$ for which all plaquettes in $\mathscr{P}^{r}$ and $\mathscr{P}^{r+1}$ within distance $10 l_{r}$ of $e$ are small field ( $S$-plaquettes). (This makes all relevant hypercubes small field, as defined before (11.2) of IV, and all relevant plaquettes small field, in the estimates to follow.) The assignment to $e, g(e, r+1)$, as given by (11.14) of IV, we write as $g^{G}(e)$ (the assignment "by gauge") and the actual isolated field assignment by $g(e)(=g(E, e))$. For $e \in \mathscr{E}_{I}^{r+1}$ one has $g(e)=g^{G}(e)$. We will want to study properties of $g^{G}(e)$, and estimate $d\left(g(e), g^{G}(e)\right)\left(\sim\left|g(e)-g^{G}(e)\right|\right)$.

Bounds on $\left|g(e)-g^{G}(e)\right|$ for SFN-Edges. We first make some definitions. We let $a_{r+1}$ and $a_{r}$ be bounds on plaquette assignments in $\mathscr{P}^{r+1}$ and $\mathscr{P}^{r}$ respectively that are in the SFN of our edge (bounds on the relevant plaquettes in $\mathscr{P}^{r+1}$ and $\mathscr{P}^{r}$ ). We consider hypercubes, $H_{i}$, in $\mathscr{L}^{r}$ in the SFN of our edge. Let $\phi_{i}$ be the associated gauge potentials on $H_{i}$, as constructed in Sect. 11 of IV. Let $H_{i}$ and $H_{j}$ intersect in their boundary portion $S_{i j}$. We then define (using the definition (11.1) of IV of $d^{g}$ )

$$
\underline{\Delta \phi}=\operatorname{Sup}_{i, j} d^{g}\left(\left.\phi_{i}\right|_{S_{i j}},\left.\phi_{j}\right|_{s_{i j}}\right) .
$$

For $i=j$ we replace $d^{g}(\cdots)$ by the greatest distance between the actual assignment to an edge of $H_{i}$ and the assignment by $\phi_{i}$. The bounds in this section will depend on $a_{r}, a_{r+1}$, and $\Delta \phi$. They will have some disappointingly large $N$ dependences. 
We distinguish between edges in $\mathscr{E}_{I}^{r+1}$, edges within a block but not in $\mathscr{E}_{I}^{r+1}$, and edges in a channel. Our final results will be summarized in (3.20)-(3.22).

a) Edges in $\mathscr{E}_{I}$. For edges in $\mathscr{E}_{I}$ we have (of course)

$$
\left|g(e)-g^{G}(e)\right|=0 \text {. }
$$

b) Edges in a block, not in $\mathscr{E}_{I}$. We consider an edge $e$ that joins two vertices $v_{1}$ and $v_{2}$ (in a block). We let $v_{0}$ be the basepoint of the block. In a gauge where all edges in $\mathscr{E}_{I}^{+{ }^{+1}}$ are assigned the identity it is easy to see (by Lemma 5.5 of III)

$$
\left|g^{\prime}(e)\right| \leqq c N a_{r+1} .
$$

The prime indicates we are in a different gauge, $g(e)$ is a gauge transformation of $g^{\prime}(e)$. We have also used Radial Property 4 of Appendix E. Inequality (3.3) follows from considering the loop $e t_{v_{0} v_{1}}^{-1} t_{v_{0} v_{2}}$. We get, undoing the gauge transformation (with $g\left(t_{v_{0}, v_{1}}\right)=g_{t_{v_{0}, v_{1}}}$ )

$$
g(e)=g\left(t_{v_{0} v_{2}}\right) g^{\prime}(e) g^{-1}\left(t_{v_{0} v_{1}}\right) .
$$

In the case all edges in $t_{v_{0} v_{2}}$ and $t_{v_{0} v_{1}}$ receive assignments from the same $H_{i}$ (in (11.14) of IV), one has

$$
g^{G}(e)=g\left(t_{v_{0} v_{2}}\right) g^{-1}\left(t_{v_{0} v_{1}}\right)
$$

and from (3.3)-(3.5) we deduce

$$
\left|g(e)-g^{G}(e)\right| \leqq c N a_{r+1} .
$$

In general we have

$$
\left|g^{G}(e)-g\left(t_{v_{0} v_{2}}\right) g^{-1}\left(t_{v_{0} v_{1}}\right)\right| \leqq c \Delta \phi
$$

and thus

$$
\left|g(e)-g^{G}(e)\right| \leqq c N a_{r+1}+c \Delta \phi .
$$

c) Channel Edges. We first state a little lemma

\section{Little Lemma.}

$$
|| g_{1} g_{2} g_{3} g_{4}|-| g_{1} g_{3} g_{2} g_{4}|| \leqq 2\left|g_{2}\right| \text {. }
$$

We now consider a plaquette containing two neighboring channel edges and two edges from each of two neighboring blocks. The channel edges

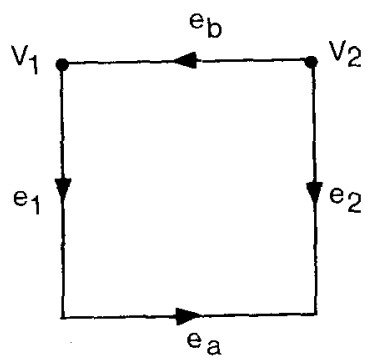

Fig. 1. 
are $e_{1}$ and $e_{2}$. We write

$$
g\left(e_{i}\right)=u_{i}^{-1} g_{i}^{\delta} u_{i} g_{i}^{G}, \quad i=1,2
$$

where

$$
u_{i}=g_{t_{v_{0} v_{i}}} \text {. }
$$

We are laying down the $g_{i}^{\delta}$, as though they were pinned to the base point of the block containing $v_{1}$ and $v_{2}$, via gauge invariant coupling. We have

$$
\left|u_{1} g_{1}^{G} g_{a}\left(g_{2}^{G}\right)^{-1} u_{2}^{-1}\left(g_{2}^{\delta}\right)^{-1} u_{2} g_{b} u_{1}^{-1} g_{1}^{\delta}\right| \leqq a_{r+1} .
$$

We have set $g_{a}=g\left(e_{a}\right)$, and likewise with $b$, and moved the $u_{1}^{-1} g_{1}^{\delta}$ from the left side to the right side of the product. We deduce from the little lemma that

$$
\left|u_{1} g_{1}^{G} g_{a}\left(g_{2}^{G}\right)^{-1} u_{2}^{-1} u_{2} g_{b} u_{1}^{-1}\left(g_{2}^{\delta}\right)^{-1} g_{1}^{\delta}\right| \leqq a_{r+1}+2\left|u_{2} g_{b} u_{1}^{-1}\right|
$$

which implies

$$
\begin{aligned}
\left|\left(g_{2}^{\delta}\right)^{-1} g_{1}^{\delta}\right| & \leqq a_{r+1}+2\left|u_{2} g_{b} u_{1}^{-1}\right|+\left|g_{1}^{G} g_{a}\left(g_{2}^{G}\right)^{-1} g_{b}\right| \\
& \leqq c N a_{r+1}+c \Delta \phi
\end{aligned}
$$

(using (3.8) and other simple observations).

Writing analogs of (3.10) for all the edges of a channel we deduce for any two edges in the same channel, $e_{i}$ and $e_{j}$,

$$
\left|g^{\delta}\left(e_{i}\right)^{-1} g^{\delta}\left(e_{j}\right)\right| \leqq c N^{2} a_{r+1}+c N \Delta \phi .
$$

We now let $\Gamma_{1}$ and $\Gamma_{2}$ be two paths whose group elements $g_{\Gamma_{1}}$ and $g_{\Gamma_{2}}$ enter into the average determining the assignments to the edge $e_{0}$ in $\mathscr{E}^{\circ r}$ "above" the channel in question. With $e_{1}$ and $e_{2}$ the channel edges in $\Gamma_{1}$ and $\Gamma_{2}$ respectively we have from (3.16) and (3.8),

$$
d\left(g_{\Gamma_{1}}, g_{\Gamma_{2}}\right) \leqq c N \Delta \phi+c N^{2} a_{r+1}
$$

and

$$
\left|g^{\delta}\left(e_{1}\right)\right| \leqq c N^{2} a_{r+1}+c N \Delta \phi+d\left(g_{\Gamma_{1}}, g\left(e_{0}\right)\right) .
$$

These two equations easily imply

$$
\left|g^{\delta}\left(e_{1}\right)\right| \leqq c N^{2} a_{r+1}+c N \Delta \phi .
$$

The fruit of this analysis is in Eqs. (3.2), (3.8), and (3.19). We collect these questions in one place:

a) For edges in $\mathscr{E}_{I}+1$,

$$
\left|g(e)-g^{G}(e)\right|=0
$$

b) For edges in blocks

$$
\left|g(e)-g^{G}(e)\right| \leqq c N a_{r+1}+c \Delta \phi .
$$

c) For edges in channels

$$
\left|g(e)-g^{G}(e)\right| \leqq c N^{2} a_{r+1}+c N \Delta \phi .
$$


(The reader may note these are similar to (E.3), (E.4), and (E.11).) The $c N \Delta \phi$ in (3.22) seems excessive to us, probably this can be improved to $c \Delta \phi$ by modifying the construction of Sect. 11 of IV. We finally note here the basic estimate

$$
\Delta \phi \leqq c a_{r} .
$$

\section{Smoothness of Local Gauge Fields}

In this section we finally face up to the task of obtaining general estimates for the edge assignments of the isolated field of a chunk. We will use material from Sect. 2 (Theorem 2.2), Sect. 3 ((3.20)-(3.23)), Sect. 11 of IV, Appendix C and Appendix D. We begin by making definitions similar to Definitions 2.1.

Definitions 4.1. Let $E$ be a chunk. We consider the isolated field of $E$, and all edge and plaquette assignments, in this section, are of this isolated field. For any point $x$ in $R^{4}$, and level $r^{\prime}$, we now define $\hat{r}_{E}\left(x, r^{\prime}\right)$ by

$$
\hat{r}_{E}\left(x, r^{\prime}\right)=\operatorname{Max}\left\{\hat{S}_{E}(x) \cap\left[0, r^{\prime}\right]\right\}
$$

with

$$
\widehat{S}_{E}(x)=\left\{r, \exists p \in \mathscr{P}^{r}, p \text { is an } L \text {-plaquette, } d(p, x) \leqq c_{0} l_{r}\right\} .
$$

Here an $L$-plaquette is understood as one of $E$. $c_{0}$ is some number such as 20 or so (which embraces all the hypercurves we deal with at a given level, near $x$ ). If $\hat{r}_{E}\left(x, r^{\prime}\right)$ is undefined we set $\hat{r}_{E}\left(x, r^{\prime}\right)=r(E)-1(r(E)$ the level of $E), \hat{r}_{E}\left(x, r^{\prime}\right)$ sort of finds the lowest level of an $L$-plaquette living above $x$, viewed as living at level $r^{\prime}$.

We now make a related definition. For any point $x$ in $R^{4}$, we define $a_{r}(x)$,

$$
a_{r}(x)=\operatorname{Max}\left\{\left|g_{\partial_{p}}\right|, \quad p \in \mathscr{P}^{r}, \quad d(p, x)<c_{0} l_{r}\right\} .
$$

The Prototype Case. We view an edge $e$ at level $r$, and let $x_{0}$ be one vertex of $e$. We assume $\hat{r}_{E}\left(x_{0}, r\right)=r(E)-1$. (This implies that we are away from the influence of gauge singularities and large gauge fields.) We assume at each level $s \leqq r$, each hypercube at distance $\leqq\left(c_{0} / 2\right) l_{s}$ from $x_{0}$ is of depth $s$. In the language of Appendix D, we are in a no-jump situation.

Theorem 4.2. In this prototype case we have the following bound for $g(e)$ :

$$
|g(e)| \leqq c N^{2} \sum_{s \leqq r}\left(\frac{l_{r}}{l_{s}}\right) a_{s}\left(x_{0}\right) .
$$

To prove this we write for each hypercube, $H_{i}$, just mentioned above, the gauge potential $\phi_{i}(x)$ as

$$
\phi_{i}(x)=\phi_{i}^{\prime}(x) \phi_{i}^{d}(x)
$$

This is (11.3) of IV. Here $\phi_{i}^{d}(x)$ is the restriction to $H_{i}$ of the potential of the hypercube containing $H_{i}$ of next larger size. (See Appendix C, Subsection 1.) If $H_{i}$ is level $s$, we deduce from (2.20)-(2.23) and Appendix $\mathrm{D}$, that $\phi_{i}^{\prime}(x)$ may be picked as satisfying

$$
l_{s} \Delta_{1}\left(\phi_{i}^{\prime}\right) \leqq c N^{2} a_{s}\left(x_{0}\right)+c N a_{s-1}\left(x_{0}\right)
$$


in the notation of Sect. 11 of IV. We are using the no-jump conditions here, see Appendix D. We have

$$
|g(e)| \leqq c N^{2} a_{r}\left(x_{0}\right)+c N a_{r-1}\left(x_{0}\right)+l_{r} \Delta_{1}\left(\phi_{a}\right)
$$

from (3.20)-(3.23) and the definition of $\Delta_{1}$ (from Sect. 11 of IV). Here $\phi_{a}$ is the gauge potential of the next larger hypercube containing the level $r$ hypercube from which $e$ takes its assignment. We have

$$
\Delta_{1}\left(\phi_{a}\right) \leqq \Delta_{1}\left(\phi_{a}^{\prime}\right)+\Delta_{1}\left(\phi_{a}^{d}\right)
$$

(by splitting differences). This extends by induction

$$
\Delta_{1}\left(\phi_{a}\right) \leqq \Delta_{1}\left(\phi_{a}^{\prime}\right)+\Delta_{1}\left(\phi_{b}^{\prime}\right)+\Delta_{1}\left(\phi_{c}^{\prime}\right)+\cdots,
$$

where $a, b, c$ label successively larger hypercubes, each containing the other. From (4.5), (4.6) and (4.8) we get

$$
\begin{aligned}
|g(e)| \leqq & c N^{2} a_{r}\left(x_{0}\right)+c N a_{r-1}\left(x_{0}\right)+l_{r} / l_{r-1}\left(c N^{2} a_{r-1}\left(x_{0}\right)+c N a_{r-2}\left(x_{0}\right)\right) \\
& +l_{r} / l_{r-2}\left(c N^{2} a_{r-2}\left(x_{0}\right)+c N a_{r-3}\left(x_{0}\right)\right)+\cdots .
\end{aligned}
$$

This is Theorem 4.2 .

The General Small Gauge Field Case. We deal with a situation as the prototype case, $\hat{r}_{E}\left(x_{0}, r\right)=r(E)-1$, but we impose no condition on depths.

Theorem 4.3. In the small gauge field case we have the bound for $g(e)$,

$$
|g(e)| \leqq c N^{2} \sum_{s \leqq r}\left(\frac{l_{r}}{l_{s}}\right)^{1-\varepsilon} a_{s}\left(x_{0}\right) .
$$

( $\varepsilon$ may be picked as small as desired by making $N$ large enough.)

Using the analysis of Appendix D, Subsect. 3, (4.5) is replaced with

$$
l_{s} \Delta_{1}\left(\phi_{i}^{\prime}\right) \leqq c N^{2} a_{s}\left(x_{0}\right)+c N a_{s-1}\left(x_{0}\right)+\operatorname{Sup}_{j \in \mathcal{N}_{i} \cup i} c l_{s} \Delta_{1}\left(\phi_{j}^{d}\right) .
$$

This replaces (4.3) with

$$
|g(e)| \leqq c N^{2} \sum_{s \leqq r}\left(\frac{l_{r}}{l_{s}}\right) a_{s}\left(x_{0}\right) c_{1}^{r-s}
$$

which readily yields the theorem.

The General Case. With $x_{0}$ one vertex of $e$, let $\hat{r}_{E}\left(x_{0}, r\right)$ be abbreviated as $\hat{r}$. Recall $r$ is the level of $e$. We define $g^{G(t)}(e)$ as the assignment to $e$ by the gauge potentials of the hypercubes of level $\hat{r}+1$.

We define $\operatorname{Disc}_{E}^{\hat{r}}\left(x_{0}\right)$ by

$$
\operatorname{Disc}_{E}^{\hat{r}}\left(x_{0}\right)=l_{\hat{r}+1} \sup _{i \neq j} \Delta_{1}\left(\left.\phi_{i} \phi_{j}^{-1}\right|_{H_{i} \cap H_{j} \cap U\left(x_{0}\right)}\right) \text {, }
$$

where $i, j$ label hypercubes at level $\hat{r}+1 . \phi_{i}$ is the gauge potential associated to $H_{i}$.

$$
U\left(x_{0}\right)=\left\{x, d\left(x, x_{0}\right)<c_{0} l_{r+1}\right\} .
$$


Theorem 4.4. In the general case

$$
\left|g(e)-g^{G(f)}(e)\right| \leqq c N^{2} \sum_{i+1 \leqq s \leqq r}\left(\frac{l_{r}}{l_{s}}\right)^{1-\varepsilon} a_{s}\left(x_{0}\right)+c\left(\frac{l_{r}}{l_{f+1}}\right)^{1-\varepsilon} \operatorname{Disc}_{E}^{f}\left(x_{0}\right)
$$

with the same statement about $\varepsilon$ as in Theorem 4.3. We note that we may use (4.15) for $\hat{r}$ any value in the range

$$
r-2 \geqq \hat{r} \geqq \hat{r}_{E}\left(x_{0}, r\right) .
$$

Some Qualitative Quantitative Bounds. From the discussion in Appendix $D$ and the bounds of the Geometric Constructions in IV we may deduce estimates on the terms in (4.15).

Discontinuity Estimate.

$$
\operatorname{Disc}_{E}^{\hat{r}}\left(x_{0}\right) \leqq c .
$$

We now define Suspicious Hypercubes in $E$. A hypercube $H$ of level $r$ is not suspicious if all $H_{i}$ of level $r$ satisfying $d\left(H_{i}, H\right)<\left(c_{0} / 2\right) l_{r}$ are small field, and all $H_{i}$ of level $r-1$ satisfying $d\left(H_{i}, H\right)<\left(c_{0} / 2\right) l_{r-1}$ are small field. Otherwise it is suspicious.

Gauge Singularity Estimate. In the notation of Theorem 4.4

$$
\left|g^{G(\hat{)})}(e)\right| \leqq c \sum_{\alpha} \frac{l_{r}}{d\left(e, x_{\alpha}\right)}+c \frac{l_{r}}{l_{r}},
$$

where $x_{\alpha}$ are the locations of the centers of gauge singularities. The number of $x_{\alpha}$ (influencing $g^{G(f)}(e)$ ) in any set $\mathscr{S} \subset R^{4}$ is

$$
\leqq c N(\mathscr{P}, \hat{r}),
$$

where $N(\mathscr{S}, \hat{r})$ is the number of suspicious hypercubes $H_{\gamma}$ of level $r_{\gamma}<\hat{r}$ and intersecting $\mathscr{S}$.

Estimating Gauge Field Smoothness. We start with an elementary estimate for unitary operators

$$
\begin{aligned}
\left|A a b^{-1} B^{-1}-C c d^{-1} D^{-1}\right| \leqq & \left|a b^{-1}-c d^{-1}\right|+\left|B^{-1} A-D^{-1} C\right| \\
& +\left|A^{-1} C\right|\left|c d^{-1}\right|+\left|A^{-1} C\right|\left|D^{-1} A\right|,
\end{aligned}
$$

where here, at slight deviation from our usual rule, we mean for a unitary operator $u$,

$$
|u|=|u-\mathbf{I d}| \text {. }
$$

The estimate follows from the identity

$$
\begin{aligned}
\left|A a b^{-1} B^{-1}-C c d^{-1} D^{-1}\right|= & \left|a b^{-1} B^{-1} A-A^{-1} C c d^{-1} D^{-1} A\right| \\
= & \mid\left(a b^{-1} B^{-1} A-c d^{-1} B^{-1} A\right)+\left(c d^{-1} B^{-1} A-c d^{-1} D^{-1} C\right) \\
& +\left[\left(c d^{-1} D^{-1} A\right),\left(A^{-1} C\right)\right] \mid .
\end{aligned}
$$

We consider parallel edges $e_{1}$ and $e_{2}$ at level $r$, with vertices $(a, b)$ and $(c, d)$ respectively. From considerations as leading to (4.6) we have

$$
\left|g\left(e_{1}\right)-g\left(e_{2}\right)\right| \leqq c N^{2}\left(a_{r}(d)+a_{r}(b)\right)+c N a_{r-1}(d)+\left|\Phi^{-1}(a) \Phi(b)-\Phi^{-1}(c) \Phi(d)\right|,
$$


assuming

$$
d\left(e_{1}, e_{2}\right)<\frac{c_{0}}{2} l_{r-1} .
$$

Here

$$
\Phi(x)=\phi_{1}^{\prime}(x) \phi_{2}^{\prime}(x) \cdots,
$$

the $\phi_{i}^{\prime}(x)$, gauge potentials of higher and higher level hypercubes as $i$ increases. We estimate the last term in (4.22) using (4.20) in an inductive manner. We use

We arrive at

$$
\phi_{j}^{d}=\phi_{j}^{\prime} \phi_{j+1}^{\prime} \cdots \text {. }
$$

$$
\begin{aligned}
& \left|\Phi^{-1}(a) \Phi(b)-\Phi^{-1}(c) \Phi(d)\right| \\
& \leqq \\
& \quad \sum_{i}\left|\phi_{i}^{\prime}(a)^{-1} \phi_{i}^{\prime}(b)-\phi_{i}^{\prime}(c)^{-1} \phi_{i}^{\prime}(d)\right| \\
& \quad+\left|\phi_{1}^{\prime}(c)^{-1} \phi_{1}^{\prime}(d)\right|\left|\phi_{1}^{d}(a) \phi_{1}^{d}(c)^{-1}\right|+\left|\phi_{1}^{d}(a) \phi_{1}^{d}(c)^{-1}\right|\left|\phi_{1}^{d}(d) \phi_{1}^{d}(a)^{-1}\right| \\
& \quad+\sum_{2}\left\{\left|\phi_{i}^{\prime}(b)^{-1} \phi_{i}^{\prime}(d)\right|\left|\phi_{i}^{d}(d) \phi_{i}^{d}(c)^{-1}\right|+\phi_{i}^{\prime}(b)^{-1} \phi_{i}^{\prime}(d)|| \phi_{i}^{\prime}(c)^{-1} \phi_{i}^{\prime}(b) \mid\right\} .
\end{aligned}
$$

This is convenient for estimation. In addition to estimates already used in this section recall (11.11) and (11.13) of IV for use in conjunction with (4.26).

To use (4.26) effectively we need sharper estimates on $\Delta_{1}\left(\phi_{i}^{\prime}\right)$ than we have so far detailed. We introduce a concept related to depth. We define the iterated depth of a level $r$ hypercube $H_{i} . r_{\text {id }}\left(H_{i}\right)$ is the minimum $r^{\prime}$ for which one can find a sequence of hypercubes

$$
H_{i}, H_{i 1}, H_{i 2}, \ldots, H_{i s}
$$

with

$$
\text { level } H_{i t}=r-t, \quad \text { level } H_{i s}=r^{\prime}
$$

(so $r^{\prime}=r-s$ ) and such that

$$
\partial H_{i} \cap \partial H_{i 1} \neq \varnothing, \quad \partial H_{i t} \cap \partial H_{i(t+1)} \neq \varnothing .
$$

Note $r_{i d}\left(H_{i}\right) \leqq r_{d}\left(H_{i}\right)\left(r_{d}\right.$ the depth). Then if $r_{i d}\left(H_{i}\right)>r_{E}\left(x_{0}, r\right)$, with $x_{0} \in H_{i}$, one has

$$
l_{r} \Delta_{1}\left(\phi_{i}^{\prime}\right) \leqq c N^{2} \sum_{r_{i d}-1 \leqq s \leqq r}\left(\frac{l_{r}}{l_{s}}\right)^{1-\varepsilon} a_{s}\left(x_{0}\right) .
$$

\section{Edge Smoothing, the Principal Principle}

It is possible that edge smoothing is not necessary for the convergence of the cluster expansion, but we find it, at the least, makes the convergence more natural to prove. Edge smoothing, as introduced in Sect. 10 of IV, is the smoothing of the isolated chunk field near the edge (boundary) of the chunk. We have required the isolated chunk field to satisfy

$$
\left|g_{\partial p}\right| \leqq c_{1} a
$$


for all $S$-plaquettes $p$, and some fixed suitable $c_{1}$. If we do not perform edge smoothing this $c_{1}$ would of necessity depend on $N$-this we do not desire. In this section we do not directly prove the possibility of edge smoothing. Rather we relate this possibility to the following property that we will prove in a later paper.

Inductive Property ER, Edge Rounding. Assume that in the inductive construction of the isolated chunk field one has been able to perform edge smoothing for all levels less than or equal $r$. Then at level $r+1$ one may modify the assignments on edges outside the chunk and on $\mathscr{E}_{A}^{r+1}$ to ensure

1. Averaging holds,

2. $\left|g_{\partial p}\right| \leqq c_{2} a$ for all $S$-plaquettes in $\mathscr{P}^{r+1}$ that are at distance $\leqq \frac{1}{2} C_{1} l_{r}$ from edges of the chunk in $\mathscr{E}^{r+1}$ (for suitable $c_{2}$ ).

So at the inductive step we are assured by this property a smoothing by modifying edge assignments outside the chunk (and on $\mathscr{E}_{A}^{\text {r+1 }}$ ), but the smoothing does not meld into the inductively constructed field at distance $\geqq \frac{1}{4} C_{1 c} l_{r}$ as required by edge smoothing. In this section we prove this is sufficient, i.e. the following theorem.

Theorem 5.1. The existence of edge rounding at each level implies the existence of edge smoothing. That is, if Inductive Property ER holds, for a given $c_{2}$, then edge smoothing is possible for $c_{1}=c_{1}\left(c_{2}\right)$.

To prove the theorem it is sufficient to show that if at a given level, $r+1$, edge rounding is possible, then at this same level, edge smoothing is possible. This we proceed to show.

We are given two configurations on $\mathscr{E}^{2 r+1}$, the configuration constructed in Sect. 10 of IV, $\left\{g^{\circ}(e)\right\}$ o standing for "original," and the edge rounded configuration $\left\{g^{\mathrm{er}}(e)\right\}$. These agree on edges of the chunk. We construct the final edge smoothed configuration in a number of steps, which we proceed to detail.

A Word of Explanation and Guidance. The edge smoothed configuration is constructed by interpolating between the edge rounded configuration near the chunk, and the original configuration at a distance from the chunk, using a smooth function to exhibit the partition (Step 3). Naturally enough one puts the edge rounded configuration in a gauge to make it as close as possible to the original configuration-here making them identical on a maximal tree (Step 1). However if one uses a single maximal tree, estimates on the difference between bond variables of the two configurations are particularly bad in the channels, so we average over choices of the maximal trees (Step 2), which trees are constructed by geometric divisions of $R^{3}$ (Step 0). After the interpolation of Step 3 averaging will no longer be satisfied, and this is corrected in Steps 4 and 5, corrected not just on the edges of $\mathscr{E}_{A}^{r+1}$, which would have led to too violent plaquette variables near $\mathscr{E}_{A}{ }_{A}^{+1}$. The details are pursued in Appendix $E$.

Step 0 . This first step is purely of geometric nature. We will divide $R^{4}$ into regions, tiles, each bounded by pieces of planes. We will consider a class of such tilings, a tiling will be labeled by $t, t \in T$. We first define primary planes. Let $B_{1}$ and $B_{2}$ be neighbouring blocks in $\mathscr{R}^{r+1}$ (separated by a channel joining them) with base points 
$v_{1}$ and $v_{2}$ in $\mathscr{E}^{r+1}$. The perpendicular bisector of the line segment joining $v_{1}$ and $v_{2}$ is a primary plane. The set of all primary planes divides space into tiles, each tile containing the vertices of $\mathscr{E}^{r+1}$ contained in some block in $\mathscr{B}^{r+1}$. We call this the primary tiling. A sub-primary plane $H$ is a plane satisfying

a) $H$ is parallel to some primary plane.

b) There is a primary plane, $H_{0}$, parallel to $H$ such that $d\left(H, H_{0}\right)=k N^{-r-1}, k$ an integer, $k<\frac{1}{5} N$.

We now take a collection of sub-primary planes with the following properties:

a) Each primary plane is within distance $\frac{1}{5} N^{-r}$ of some member of this set parallel to it.

b) Two parallel planes of the collection are at distance $>\frac{3}{5} N^{-r}$ from each other.

This tilings we will consider will be in 1-1 correspondence with such a collection of sub-primary planes. We will say a tiling is associated to a proper collection of sub-primary planes.

We now proceed to define the tiling, $t$, associated to a given proper collection of sub-primary planes. We do not merely take the division of $R^{4}$ these planes yield, since we will want the tiling to be as the primary tiling within the chunk. We define a core region in $R^{4}$ as the union of those tiles of the primary tiling that are at distance $<10 N^{-r}$ from the set of edges $\mathscr{E}^{r+1} \cap E$. The core region is tiled as by the primary tiling. Tiles, obtained by the division of $R^{4}$ by the proper collection of sub-primary planes, that are at distance greater than $\frac{1}{2} N^{-r}$ from the core region are also part of our tiling. We have tiled $R^{4}$ except for a zone surrounding the core region. We now divide the zone into tiles. The zone is tiled by distorted hypercubes, objects with 8 hyperplane (hyper) faces; but these faces will not be required to be naturally parallel or perpendicular. Rather they will be determined by the 16 vertices of the distorted hypercubes. These vertices will be contained either in the vertices of the hypercubes in the boundary of the core region, or in the vertices of the hypercubes in the boundary of the region built up of hypercubes determined by the subprimary planes. (Vertices are located on either the inner or outer boundary of the zone, as vertices of the rectangular tiles already constructed.) It is clear which vertices to select for each distorted cube, ones "minimizing the distortion."

In fact there are an infinite number of tilings $t$ as we have defined them. But they may be divided into a finite number of equivalence classes so that tilings that are members of the same equivalence class have the same contribution to the construction to follow. (The tiling of space far enough away from the core region, $>\frac{1}{4} C_{1 c} l_{r^{\prime}}$ is irrelevant.) From now on we implicitly work with these equivalence classes.

Step 1. We are given a tiling $t$. Each tile contains a single base point within it. A radial maximal tree centered at the base point is constructed within each tile. (Each vertex in $\mathscr{V}^{r+1}$ is in one and only one maximal tree. Points on the boundary of neighboring tiles lie on the maximal tree of one of the tiles sharing the boundary. Further properties of the maximal trees will be given later.) We perform a gauge transformation on the field configuration $\left\{g^{\mathrm{er}}(e)\right\}$ so that assignments of $\left\{g^{o}(e)\right\}$ 
and the gauge transformed field $\left\{g^{\text {ert }}(e)\right\}$ agree on edges of the maximal trees associated to the tiling $t$.

Step 2. We find the modified edge rounding assignments on $\mathscr{E}^{\mathrm{r}+1}, g^{\mathrm{mer}}(e)$, by averaging the assignments on $\mathscr{E}^{e r+1}, g^{\operatorname{mer}}(e)$, over tilings, $t$. That is we minimize

$$
\sum_{i} d^{2}\left(g^{\mathrm{mer}}(e), g^{\mathrm{ert}}(e)\right)
$$

Step 3. We now have two configurations on $\mathscr{E}^{\text {rr+1 }}$, the original assignments of Sect. 10 of IV, and our modified edge rounded assignments. These assign to an edge $e$ values $g^{o}(e)$ and $g^{\text {mer }}(e)$ respectively. We find a function $\chi_{E}(x)$ on $R^{4}$ that satisfies

a) $o \leqq \chi_{E}(x) \leqq 1$,

b) $\left|D \chi_{E}\right| \leqq c N^{r}$

c) $\chi_{E}(x)=1$ if the distance between $x$ and edges in $\mathscr{E}^{\mathscr{C}+1} \cap E$ is $\geqq \frac{3}{16} C_{1 c} l_{r}$,

d) $\chi_{E}(x)=0$ if the distance between $x$ and edges in $\mathscr{E}^{\circ+1} \cap E$ is $\leqq \frac{1}{16} C_{1 c} l_{r}$.

We now take as our assignment on $\mathscr{E}^{r+1}$ for edge $e, g^{3}(e)$, defined by minimizing

$$
\chi_{E}(\hat{e}) d^{2}\left(g^{3}(e), g^{o}(e)\right)+\left(1-\chi_{E}(\hat{e})\right) d^{2}\left(g^{3}(e), g^{\text {mer }}(e)\right) .
$$

Step 4 . We now basically construct a localized form of an averaging correction mode. To each edge $e$ we associate a vector function on $R^{4}, \chi_{e}(x)$, as given by (2.1) and (2.2) of $I$. We find $C^{\infty}$ vector valued functions $\xi_{e}(x)$ with the properties:

a) The support of $\xi_{e}(x)$ lies within the support of $\chi_{e}(x)$.

b) $\int \chi_{e}(x) \cdot \xi_{e^{\prime}}(x)$ is 1 if $e^{\prime}=e$; and is zero if $e \neq e^{\prime}, e, e^{\prime}$ at the same level.

c) The functions $\xi_{e}(x)$, for different $e$, are all scalings, rotations, and translations of a single function.

We now select a single bond $e$ at level $r$. For plaquette $p$ at level $r+1$ we find plaquette assignments from $\xi_{e^{\prime}}$ as

$$
A_{\partial p}=\int \chi_{p}(x) \cdot \xi_{e}(x) .
$$

We consider the configuration on $\mathscr{E}^{\text {r }}$ with assignment 1 to edge $e$ and zero to all other edges. We set $A(e)=0$ for all $e \in \mathscr{E}_{I}^{\mathfrak{r}+1}$. Now we use the plaquette assignments of (5.8) to determine a unique configuration on $\mathscr{E}^{r+1}$, satisfying averaging with the just specified $r$ level configuration. (The configuration on $\mathscr{E}^{r+1}$ yields the plaquette assignments of (5.8), and averages to yield the just specified configuration on $\mathscr{E}^{r}$.)

Step 5. We consider the configuration on $\mathscr{E}^{r+1}$ at the end of Step 3 (the $g^{3}(e)$ ), it will not necessarily average to the correct assignments on $\mathscr{E}$. We modify the assignments on $\mathscr{E}^{\mathscr{C}+1}$ by (in any order) using gauge invariant coupling and adding the truncated averaging correction modes of Step 4 (pinned as ordinary average correction modes) with amplitudes chosen to give a combined field that, to linear order, averages to the correct field in $\mathscr{E}^{\text {r }}$. (Note, this procedure does not, as when one adds a usual average correction mode, exactly fix the averaging error. We are not modifying $\mathscr{E}^{\mathscr{r}+1}$ on a single edge to exactly satisfy averaging. Presumably we could modify our present procedure slightly to make it satisfy averaging exactly.) 
Step 6. Modify the configuration on $\mathscr{E}^{r+1}$ on edges of $\mathscr{E}_{A}^{r+1}$ to exactly average to configuration on $\mathscr{E}$.

Note. Once the edge smoothed configuration on $\mathscr{E}^{r+1}$ is constructed, it is returned to the gauge specified in Sect. 11 of IV.

\section{Appendix A. A Postscript to $I$}

In this Appendix we are in the set-up of $I$, studying the plaquette assignments of a continuum field minimizing the continuum action, for the Abelian theory, with plaquette assignments specified at length scale $L$, level $r$.

Lemma A.1. Plaquette-Plaquette fall off Estimates:

$$
\left|A_{\partial p}\right| \leqq c\left(\frac{l}{L}\right)^{2} \sum_{P \in \mathscr{P}^{\prime}}\left|A_{\partial P}\right| e^{-\gamma^{\prime} d(p, P) / L} .
$$

Here $p$ is at length scale $l$, the $P$ at length scale $L . \gamma^{\prime}$ is any number, $\gamma^{\prime}<\gamma(\gamma$ as in $I$ ). Now let $p_{1}$ and $p_{2}$ be parallel (oriented) plaquettes at length scale $l$,

$$
\left|A_{\partial p_{1}}-A_{\partial p_{2}}\right| \leqq c_{\varepsilon}\left(d\left(p_{1}, p_{2}\right)\right)^{1-\varepsilon} \frac{l^{2}}{L^{3-\varepsilon}} \sum_{P \in \mathscr{P} \mathscr{P}^{r}} e^{-\gamma^{\prime} d\left(p_{1}, P\right) / L}\left|A_{\partial P}\right|
$$

for each $\varepsilon>0$, where $d\left(p_{1}, p_{2}\right)$ is measured between corresponding vertices and

$$
d\left(p_{1}, p_{2}\right)<c L .
$$

It may seem that these results should follow immediately from the estimates in $I$, but we require a small additional argument. We find a vertex in the length scale $L$ lattice, i.e. in $\mathscr{V}^{r}$, that is closest to $p$ in (A.1) or $p_{1}$ in (A.2). We now construct the field, in the lattice $\mathscr{L}^{r}$, in a radial axial gauge radiating out from this vertex (using the universal radial tree). Of course this does not change plaquette assignments. Edge assignments are easily bounded in terms of plaquette bounds, (using Lemma 5.5 of III) bounds that, loosely speaking, grow as some power of distance in the radial direction. Using these bounds, and Estimates 0.6 and 0.7 of $I$, the lemma follows.

\section{Appendix B. Necessity of Gauge Singularities}

We construct a field configuration on the unit lattice in $R^{4}$ that is associated to a pure gauge continuum $A_{\mu}(x)$, with a point singularity, and fall off (to zero) at infinity; but which cannot be associated to any $A_{\mu}^{\prime}(x)$, that is pure gauge and everywhere continuous, and falls off to zero at infinity.

We work with a gauge group $G$, a simple compact non-abelian Lie group. (This will imply $\pi_{3}(G)$ is not trivial.) We denote by $\mathscr{V}$ the vertices of our unit lattice, a set of points in $R^{4}$. We identify $S^{3}$ with the standard sphere $\Sigma x_{i}^{2}=1$, and let

$$
f: S^{3} \rightarrow G
$$


be a continuously differentiable map that is not homotopically trivial. We pick a point $a \in R^{4}$ that is not in $\mathscr{V}$, and define

$$
\Lambda(x)=f\left(\frac{x-a}{|x-a|}\right)
$$

$\Lambda(x)$, a gauge transformation, defines a gauge field

$$
A_{\mu}(x)=\Lambda^{-1}(x) \partial_{\mu} \Lambda(x)
$$

and likewise, an associated field on the lattice. This lattice field assigns to edge $e=\overrightarrow{a b}$ the group element $\Lambda^{-1}(a) \Lambda(b) . A_{\mu}(x)$ is continuously differentiable, except at a, where it is discontinuous, and falls off to zero at infinity.

We now assume the existence of a pure gauge continuous $A_{\mu}^{\prime}(x)$, that falls off to zero at infinity, and is associated to the same lattice configuration. We will deduce a contradiction. (We interpret fall off at infinity to mean fall off uniform over spheres centered at the origin.)

Since $A_{\mu}^{\prime}(x)$ is pure gauge, and gives the same lattice field as $A_{\mu}(x)$, we can find a continuous gauge transformation $\Lambda^{\prime}(x)$ satisfying

$$
A_{\mu}^{\prime}(x)=\Lambda^{\prime-1}(x) \hat{\partial}_{\mu} \Lambda^{\prime}(x)
$$

and

$$
\Lambda^{\prime}(x)=\Lambda(x), \quad x \in \mathscr{V} .
$$

Given any $\varepsilon>0$ and any $R_{0}$ we can find an $R=R\left(\varepsilon, R_{0}\right)$ such that $R>R_{0}$, and

$$
\left|A_{\mu}^{\prime}(x)\right|<\varepsilon, \quad\left|A_{\mu}(x)\right|<\varepsilon
$$

for all $x$ within distance 2 of the sphere of radius $R, S_{R^{\prime}}$ centered at the origin.

We let $x$ be a point in $S_{R}$. We wish to bound

$$
\left|\Lambda(x)-\Lambda^{\prime}(x)\right| \text {. }
$$

(We work with a particular faithful unitary representation of $G$, and let bars indicate operator norms.) We note there is a point $\bar{x} \in \mathscr{V}$ within distance 2 of $x$, and proceed as follows:

$$
\left|\Lambda(x)-\Lambda^{\prime}(x)\right| \leqq|\Lambda(x)-\Lambda(\bar{x})|+\left|\Lambda^{\prime}(x)-\Lambda^{\prime}(\bar{x})\right|,
$$

since $\Lambda(\bar{x})=\Lambda^{\prime}(\bar{x})$. We also have

$$
|\Lambda(x)-\Lambda(\bar{x})| \leqq \int_{x}^{\bar{x}}|A(x)| d x \leqq 2 \varepsilon
$$

and a similar bound for $\Lambda^{\prime \prime}$. Thus we get

$$
\left|\Lambda(x)-\Lambda^{\prime}(x)\right| \leqq 4 \varepsilon \text { on } S_{R} .
$$

This implies, if $\varepsilon$ is small enough, that $\Lambda(x)$ and $\Lambda^{\prime}(x)$ give homotopic maps of $S_{R}$ into $G$, homotopically nontrivial maps if $R$ is large enough. This is in contradiction with the assumed continuity of $\Lambda^{\prime}(x)$ over all $R^{4}$. 


\section{Appendix C. Clarifications, Additions, Modifications, Corrections to Earlier Papers}

1. Use of Depth in Sect. 11 of IV. The concept will still be of use to us, but we have decided not to employ it in (11.3) of IV. Modify the sentence previous to this equation so that $H_{\alpha}$ is the level $(r-1)$ hypercube containing $H_{i}$.

2. Radial Trees. In addition to the treatment of radial maximal trees in Sect. 5.4, B) of III, we find amplifications as follows.

a) In the first paragraph of Appendix E.

b) We assume the straight line path realizing the shortest distance between nearest neighbor base points is built up of portions of the maximal trees (and a channel edge). (This is used in the argument surrounding (E.11) - but this is not necessary for this prupose.)

c) In the first paragraph of Sect. 3 .

3. Averaging in the Isolated Field of a Chunk. In our formalism each compatibility requirement between lattice assignments on different lattices is realized as a requirement on the assignment to an averaging edge. In developing this isolated chunk field, for averaging edges outside the chunk we take each such assignment to be consistent with pure small field averaging - without the modifications given in III. Thus rule was also implicitly followed for the mode field isolated assignments (there all averaging edge assignments chosen by pure small field averaging).

\section{Appendix D. Expansion of Sect. 11 of IV}

In this Appendix we make more explicit the application of the mathematical theorems of Appendix A of IV to the constructions of Sect. 11 therein. Indeed the reader may feel that the constructions of Sect. 11 of IV were themselves not presented sufficiently precisely, and we further amplify their description. We first study the invariant distance $d^{g}$ of (11.1) of IV, obtaining some simple but elegant general results.

1. Abstract Properties of $d^{g}$. Let $G$ be a group with an invariant distance $d$ on it. Thus $d\left(g_{1}, g_{2}\right)=d\left(a g_{1} b, a g_{2} b\right)$. We consider two indexed sets of group elements $\left\{g_{i}\right\}$, $\left\{h_{i}\right\} \quad i \in \mathscr{S}$ and define (the definition of (11.1) of IV in slightly different notation)

$$
d_{\mathscr{S}}^{g}\left(\left\{g_{i}\right\},\left\{h_{i}\right\}\right)=d^{g}\left(\left\{g_{i}\right\},\left\{h_{i}\right\}\right)=\operatorname{InfSup}_{u} d\left(g_{i}, u h_{i}\right) .
$$

For the same sets of elements we may restrict $\mathscr{S}$ to a subset $\mathscr{T} \subset \mathscr{S}$, and obtain the absolutely trivial result

The Subset Lemma. Let $\mathscr{T} \subset \mathscr{S}$, then

$$
d_{\mathscr{T}}^{g}\left(\left\{g_{i}\right\},\left\{h_{i}\right\}\right) \leqq d_{\mathscr{Y}}^{g}\left(\left\{g_{i}\right\},\left\{h_{i}\right\}\right) .
$$

We proceed to a more interesting, less trivial result.

The Non-empty Intersection Lemma. Let $\mathscr{A} \subset \mathscr{S}, \mathscr{B} \subset \mathscr{S}, \mathscr{A} \cap \mathscr{B} \neq \varnothing$. We for now 
abbreviate $d_{\mathscr{F}}^{g}\left(\left\{g_{i}\right\},\left\{h_{i}\right\}\right)$ by $d_{\mathscr{Y}}^{g}$ for any subset of $\mathscr{S}$. Then

$$
d_{\mathscr{S A} U \mathscr{S}}^{g} \leqq 2 \operatorname{Max}\left\{d_{\mathscr{S}}^{g}, d_{\mathscr{G}}^{g}\right\} \text {. }
$$

We turn to proving this lemma. We note the following useful equivalence

$$
d_{\mathscr{T}}^{g}\left(\left\{g_{i}\right\},\left\{h_{i}\right\}\right)<d \Leftrightarrow \exists u \ni \text { for all } i, \quad d\left(u, g_{i} h_{i}^{-1}\right)<d .
$$

Now let 0 be in $\mathscr{A} \cap \mathscr{B}$. We note from (D.4) there follows

$$
\begin{array}{ll}
d\left(g_{0} h_{0}^{-1}, g_{i} h_{i}^{-1}\right)<2\left(d_{\mathscr{A}}^{g}+\varepsilon\right), & i \in \mathscr{A}, \\
d\left(g_{0} h_{0}^{-1}, g_{i} h_{i}^{-1}\right)<2\left(d_{\mathscr{B}}^{g}+\varepsilon\right), & i \in \mathscr{B},
\end{array}
$$

for any $\varepsilon>0$. This implies

$$
d\left(g_{0} h_{0}^{-1}, g_{i} h_{i}^{-1}\right)<2\left(\operatorname{Max}\left\{d_{\mathscr{A}}^{g}, d_{\mathscr{B}}^{g}\right\}+\varepsilon\right), \quad i \in \mathscr{A} \cup \mathscr{B},
$$

which is the lemma.

We turn to a transitive property of $d^{g}$.

The Triangle Lemma. Let $\left\{g_{i}\right\},\left\{h_{i}\right\},\left\{s_{i}\right\}, i \in \mathscr{S}$ be three sets of similarly indexed group elements. Then

$$
d_{\mathscr{S}}^{g}\left(\left\{g_{i}\right\},\left\{s_{i}\right\}\right) \leqq d_{\mathscr{S}}^{g}\left(\left\{g_{i}\right\},\left\{h_{i}\right\}\right)+d_{\mathscr{S}}^{g}\left(\left\{h_{i}\right\},\left\{s_{i}\right\}\right)
$$

To prove this, using (D.4), we note

$$
\begin{aligned}
d\left(\left(g_{i} h_{i}^{-1}\right)\left(h_{i} s_{i}^{-1}\right), u_{1} u_{2}\right) & \leqq d\left(\left(g_{i} h_{i}^{-1}\right)\left(h_{i} s_{i}^{-1}\right), u_{1}\left(h_{i} s_{i}^{-1}\right)\right)+d\left(u_{1}\left(h_{i} s_{i}^{-1}\right), u_{1} u_{2}\right) \\
& \leqq d\left(g_{i} h_{i}^{-1}, u_{1}\right)+d\left(h_{i} s_{i}^{-1}, u_{2}\right) .
\end{aligned}
$$

This immediately yields the triangle lemma.

2. The Distance $d^{g}$ Between the Gauge Potentials of Neighboring Hypercubes Along Their Intersection. Initially the gauge potentials are defined only on the vertices of each hypercube. Let $H_{i}$ be a hypercube that is not large field (see Sect. 11 of IV, after (11.1)). Let $\left\{H_{j}\right\}, j \in \mathscr{N}_{i}$, be the set of hypercubes of the same level that are not large field and have non-zero intersection with $H_{i}$, its neighbors. We define

$$
\Delta(i)=\underset{j \in \mathcal{H}_{i}}{\operatorname{Max}_{i}} d^{g}\left(\left.\phi_{i}\right|_{H_{i} \cap H_{j}},\left.\phi_{j}\right|_{H_{i} \cap H_{j}}\right) \text {. }
$$

Here the $\phi_{i}, \phi_{j}$ need only be defined on the vertices of $H_{i}$. We claim, at the end of the process of extending the $\phi_{i}$ and $\phi_{j}$ to the full hypercubes, one has

$$
d^{g}\left(\left.{ }^{e} \phi_{i}\right|_{H_{i} \cap H_{j}},\left.{ }^{e} \phi_{j}\right|_{H_{i} \cap H_{j}}\right)<c \Delta(i)
$$

for all $j \in \mathcal{N}_{i}$. This implies readily (3.23).

To see (D.10) we note that the extensions are achieved by performing a finite number (independent of $N$ ) of Geometric. Constructions 1-4 of Sect. 11 of IV. (Geometric Constructions 5 and 6 are not now relevant.) It is only the application of Constructions 2 or 4 that may change the $d^{a}$ between hypercubes. Suppose an application of one of these two constructions extends the mapping $\phi_{\alpha}$ on $H_{\alpha}$ from $\partial D \subset H_{\alpha}$ to $D \subset H_{\alpha}$. $\phi_{\beta}$ on $H_{\beta}$ already is defined on $D \subset H_{\beta}$. The extension of $\phi_{\alpha}$ is done by matching as well as possible on $\partial D$ and extending to $D$ obtaining 
estimates (11.5) of IV. (This may clarify the construction process of Sect. 11 of IV.) Using D.2, D.3, and D.7 (we need all three of these), and with some thought, we deduce (D.10).

3. $\Delta_{1}$ of $\phi_{i}$, the Gauge Field on Hypercube, $H_{i}$. We recall the separation of $\phi_{i}$ in (11.3) of IV,

$$
\phi_{i}=\phi_{i}^{\prime} \phi_{i}^{d}
$$

and note, from 1) in Appendix $\mathrm{C}$, that $\phi_{i}^{d}$ is the gauge field of the next smallest hypercube containing $H_{i}$, restricted to $H_{i}$. In the case that hypercubes near $H_{i}$ and of the same level or one level higher are small field, we may read off an estimate for $\Delta_{1}\left(\phi_{i}^{\prime}\right)$ from (3.20)-(3.22) - where here we view $\phi_{i}^{\prime}$ as restricted to the vertices of $H_{i}$. That is

$$
l_{r} \Delta_{1}\left(\phi_{i}^{\prime}\right) \leqq c N^{2} a_{r}+c N a_{r-1},
$$

where $H_{i}$ is level $r$; and $a_{r}, a_{r-1}$ may be taken as $a_{r}\left(x_{0}\right), a_{r-1}\left(x_{0}\right)$ for $x_{0}$ a vertex of $H_{i}$ (in the language of (4.2)). Again, in (D.12) $\phi_{i}^{\prime}$ is viewed as living only on the vertices of $H_{i}$.

In the process of using Geometric Constructions 1 and 3 the bound on $\Delta_{1}\left(\phi_{i}^{\prime}\right)$ is at most multiplied by a constant (see (11.4) and (11.8) of IV). Let us consider using Geometric Constructions 2 and 4. One has already defined $\phi_{\beta}^{\prime}$ of $H_{\beta}$ on $D \subset H_{\beta}$. We are extending $\phi_{\alpha}$ on $H_{\alpha}$ from $\partial D \subset H_{\alpha}$ to $D \subset H_{\alpha}$. (This is the notation of the last subsection.) We carry out this construction trying to match $\phi_{\alpha}^{\prime} \phi_{\alpha}^{d}$ with $\phi_{\beta}^{\prime} \phi_{\beta}^{d}$. This is the same as matching $\phi_{\alpha}^{\prime}$ with $\phi_{\beta}^{\prime}\left(\phi_{\beta}^{d}\left(\phi_{\alpha}^{d}\right)^{-1}\right)$. If $\phi_{\beta}^{d}=\phi_{\alpha}^{d}$ on the intersection $H_{\alpha} \cap H_{\beta}$ we are in a no-jump situation. This will happen if $H_{\alpha}$ is of depth $r\left(=r\left(H_{z}\right)\right)$. This is language from Sect. 11 of IV. If all such matchings are no-jump (the hypercubes we deal with at level $r$ all are in the interior of the same hypercube at the next higher level) then we may deduce from (11.6) of IV, and (D.12) that

$$
l_{r} \Delta_{1}\left(\phi_{i}^{\prime}\right) \leqq c N^{2} a_{r}\left(x_{0}\right)+c N a_{r-1}\left(x_{0}\right) .
$$

If there are jumps possible at the interfaces of $H_{i}$ and its neighbors, we must modify (D.13).

$$
l_{r} \Delta_{1}\left(\phi_{i}^{\prime}\right) \leqq c N^{2} a_{r}\left(x_{0}\right)+c N a_{r-1}\left(x_{0}\right)+c \operatorname{Sup}_{j \in \mathscr{N}_{i}} l_{r} \Delta_{1}\left(\left.\phi_{i}^{d}\left(\phi_{j}^{d}\right)^{-1}\right|_{H_{i} \cap H_{j}}\right),
$$

$\mathscr{N}_{i}$ is a notation of the last subsection. Note that on the left sides of (D.13) and (D.14) $\phi_{i}^{\prime}$ is viewed as defined on $\partial H_{i}$. Estimates on Geometric Constructions 5 and 6 determine bounds for $\Delta_{1}\left(\phi_{i}^{\prime}\right)$ in all $H_{i}$, by either (11.11) or (11.13) of IV.

We note that if $\phi_{i}^{d}=\phi_{i}^{j} \phi_{i}^{n j}, \phi_{j}^{d}=\phi_{j}^{j} \phi_{j}^{n j}$ with $\phi_{i}^{n j}=\left.\phi_{j}^{n j}\right|_{H_{j} \cap H_{j}}$, then

$$
\Delta_{1}\left(\left.\phi_{i}^{d}\left(\phi_{j}^{d}\right)^{-1}\right|_{H_{i} \cap H_{j}}\right) \leqq \Delta_{1}\left(\phi_{i}^{j}\right)+\Delta_{1}\left(\phi_{j}^{j}\right) \text {. }
$$

Here the superscripts stand for jump, and no-jump respectively.

\section{Appendix E. Some Estimates for Edge Smoothing}

We first note an additional property of radial trees as constructed in III (at unit scale). 
Radial Property 4. Let $p_{1}$ and $p_{2}$ be two neighboring points $\left(d\left(p_{1}, p_{2}\right) \leqq c\right)$, and consider the closed loop consisting of two paths along the maximal tree from the origin to $p_{1}$ and $p_{2}$ respectively, and a path from $p_{1}$ to $p_{2}$ of length $\leqq c$. Then this closed path may be contracted to a point by a number of elementary homotopies, $N_{e^{\prime}}$ that satisfies

$$
N_{e} \leqq c+c\left|p_{1}\right| \cdot
$$

We wish to estimate

$$
\delta(e) \equiv d\left(g^{o}(e), g^{\text {ert }}(e)\right)
$$

for edges $e$ in $\mathscr{E}^{r+1}$. For $e$ an edge of one of the maximal trees associated to the tiling $t$ we have

$$
\delta(e)=0 .
$$

If the two vertices in $\partial e$ belong to the same maximal tree, we may estimate $\delta(e)$, using a gauge in which the assignments to the maximal tree are trivial, and we find (from Lemma 5.5 of III, and (E.1))

$$
\delta(e) \leqq c N a .
$$

$\left(\delta(e)\right.$ is a gauge invariant quantity-when $\left\{g^{a}(e)\right\}$ and $\left\{g^{\text {ert }}(e)\right\}$ experience the same gauge transformations.)

We now let $e_{1}$ and $e_{2}$ be edges that join the same two maximal trees of the tiling in same orientation (in the same "channel" of the tiling), then similarly to the above we find

$$
d\left(g^{\text {er1 }}\left(e_{1}\right), g^{\text {ert }}\left(e_{2}\right)\right) \leqq c N^{2} a
$$

and

$$
d\left(g^{o}\left(e_{1}\right), g^{o}\left(e_{2}\right)\right) \leqq c N^{2} a
$$

(We could improve this last estimate.) Let $\Gamma_{1}$ and $\Gamma_{2}$ be two paths in $\mathscr{L}^{r+1}$ entering the averaging of contributions to an edge $e$ in $\mathscr{E}^{2}$. Then we have easily (in the region outside the chunk)

$$
d\left(g_{\Gamma_{1}}^{o}, g_{\Gamma_{2}}^{o}\right) \leqq c N^{2} a
$$

and

$$
d\left(g_{\Gamma_{1}}^{\mathrm{ert}}, g_{\Gamma_{2}}^{\mathrm{ert}}\right) \leqq c N^{2} a
$$

(where again estimate (E.7) can be improved). Of course we have

$$
\mathrm{Av}_{\Gamma} g_{\Gamma}^{o}=\mathrm{Av}_{\Gamma} g_{\Gamma}^{\mathrm{ert}}
$$

We deduce from (E.7)-(E.9) that

$$
d\left(g_{\Gamma}^{\mathrm{ert}}, g_{\Gamma}^{o}\right) \leqq c N^{2} a
$$

Together our sequence of estimates now enable us to deduce for a "channel" edge $e$,

$$
\delta(e) \leqq c N^{2} a .
$$


(We first deduce this for the "channel" edge on the line joining base points, and then thereafter at other "channel" edges.) The estimates (E.3), (E.4) and (E.11) complete this study of the difference of edge assignments between $g^{o}$ and $g^{\text {ert }}$.

We next deduce

$$
d\left(g^{\operatorname{mer}}(e), g^{o}(e)\right) \leqq c N a
$$

for $e$ in $\mathscr{E}^{\text {r+1 }}$. This follows from the fact that the estimates (E.3) and (E.4) hold for $\sim c N^{4}$ terms in the average determining $g^{\text {mer }}(e)$ and the estimate (E.11) for $\sim c N^{3}$ terms. (This argument is questionable near the zone surrounding the core, but (E.12) is not needed in this region.)

From the bounds on $g_{\partial p}^{o}$ and $g_{\partial p}^{\text {mer }}$, and from (E.12), we deduce

$$
\left|g_{\partial p}^{3}\right| \leqq c a \text {. }
$$

(If we had settled for $d\left(g^{\text {mer }}(e), g^{o}(e)\right) \leqq c N^{2} a$, then one would have had $\left|g_{\partial p}^{3}\right| \leqq$ $\mathrm{cNa}$; this is the reason we average over tilings.)

From (E.12) (and the construction of Step 3) we deduce the averaging error at a bond $e$ in $\mathscr{E}$, from the assignments of $\left\{g^{3}(e)\right\}$, to be in error by

$$
\leqq c N^{2} a \text {. }
$$

The construction of Steps $4 \& 5$ will correct this error to within

$$
\leqq f(N) a^{2} \text {, }
$$

which error is corrected in Step 6. Step 5 changes plaquette values by

$$
\leqq c a,
$$

and Step 6 by

$$
\leqq f(N) a^{2}
$$

\section{References}

1. Federbush, P.: A phase cell approach to Yang-Mills theory. I. Modes, lattice-continuum duality. Commun. Math. Phys. 107, 319-329 (1986)

2. Federbush, P., Williamson, C.: A phase cell approach to Yang-Mills theory. II. Analysis of a mode. J. Math. Phys. 28, 1416-1419 (1987)

3. Federbush, P.: A phase cell approach to Yang-Mills theory. III. Local stability, modified renormalization group transformation. Commun. Math. Phys. 110, 293-309 (1987)

4. Federbush, P.: A phase cell approach to Yang-Mills theory. IV. The choice of variables. Commun. Math. Phys. 114, 317-343 (1988)

5. Federbush, P.: A phase cell approach to Yang-Mills theory. VI. Non-abelian lattice-continuum duality. Ann. l'Inst. Henri Poincaré 47, 17-23 (1987)

Communicated by K. Gawedzki

Received May 8, 1988; in revised form May 30, 1989 